\title{
Le batardeau à aiguilles du barrage de Saint-Pierre-de-Bœuf sur le Rhône
}

\author{
R. Pinatel, R. Bichet
}

Compagnie Nationale du Rhône

\begin{abstract}
L'aménagement du Rhône entre la frontière suisse et la mer est constitué actuellement de 19 aménagements hydroélectriques en service dont 6 sur le Haut-Rhône et 13 sur le Bas-Rhône au Sud de Lyon. Ils comprennent en tout 21 barrages car les chutes de Bourg-les-Valence et d'Avignon en possèdent chacune deux. Mis à part Génissiat, qui est un ouvrage poids de moyenne chute, tous les aménagements sont de basse chute et comprennent en général un barrage mobile, une dérivation, une usine hydroélectrique et une écluse. Les barrages permettent de créer la chute nécessaire au fonctionnement des turbines et assurent le rétablissement du débit dans le Rhône court-circuité lors des crues ou en cas d'incident à l'usine.
\end{abstract}

\section{Evolution dans la conception des barrages mobiles du Rhône}

Les premiers barrages mobiles construits par la Compagnie Nationale du Rhône étaient largement dimensionnés au regard des énergies linéiques dissipées et des dimensions dans le sens amont-aval. Ils n'ont donné lieu à aucun problème d'entretien dans les zones aval, sauf quelques recharges faciles du tapis en enrochements. Le parti pris de ne mettre aucune disposition de batardement aval était ainsi parfaitement justifié, d'autant que les parties nobles de l'ouvrage au voisinage des batardeaux amont étaient peu noyées et pouvaient s'entretenir par des moyens relativement simples.

Par la suite, dans un souci d'économie, le profil des barrages a été progressivement modifié, la cuvette de dissipation d'énergie des premiers ouvrages étant remplacée par une dent d'environ $1 \mathrm{~m}$ de hauteur, derrière un radier plongeant (dent partiellement ou totalement blindée). De même, les dimensions amont-aval se réduisaient et l'énergie linéique dissipée augmentait. Dans ces conditions, un accroissement des entretiens à l'aval était prévisible, ce qui a conduit à sceller des pièces fixes (dans des rainures) en prévision d'un batardement éventuel.

Parallèlement, les revêtements de protection des radiers et des piles étaient l'objet de modification. Dans les premiers barrages cette protection était assurée par des granits durs scellés dans le radier et la base des piles (fig. 1). Progressivement, seules les arêtes vives ont été protégées par des granits, les autres surfaces planes étant revêtues de ciment-corindon (fig. 2). Enfin, dans une dernière étape, les granits ont complètement disparu, la protection étant entièrement assurée par un revêtement anti-usure en brai-époxy-corindon et quelques blindages appliqués sur les zones très sensibles (redans, rainures, bas de piles, ...) (fig. 3).

\section{Apparition de désordres sur des barrages de conception récente}

L'augmentation de l'énergie linéique à dissiper à travers les passes et certaines compositions du sol d'assise ont entraîné une plus grande sollicitation des zones soumises à usure (rainure à batardeaux, redans aval, ...). Certains barrages ont donné lieu à des incidents sérieux qui demandaient des travaux d'entretien importants à l'aval, ne pouvant plus être réalisés par des interventions légères sous l'eau mais par des opérations lourdes à l'abri d'un batardement.

La nécessité de pouvoir examiner, entretenir et réparer à sec ces ouvrages, a conduit la Compagnie Nationale du Rhône à mettre au point un dispositif de batardement aval.

\section{Cas particulier du barrage de Péage de Roussillon}

C'est ce barrage qui a été le plus exposé et qui a dû faire l'objet des premières interventions importantes. Les culées et les piles possédaient à l'aval, des rainures à batardeaux qui devaient permettre le batardement d'une passe quelconque, en utilisant les éléments des batardeaux amont, leur mise en place étant réalisée par l'intermédiaire d'un outillage mis en œuvre à partir des ponts amont ou d'un ponton flottant à l'aval, dont la fourniture n'était toutefois pas prévue à l'origine. Le barrage comprend également un redan à l'aval de chacune des passes, ainsi que des murs en aile à l'amont et à l'aval. C'est enfin le premier barrage du Rhône à ne comporter aucun granit, et où tous les radiers ont reçu un revêtement anti-usure en brai-époxy- 


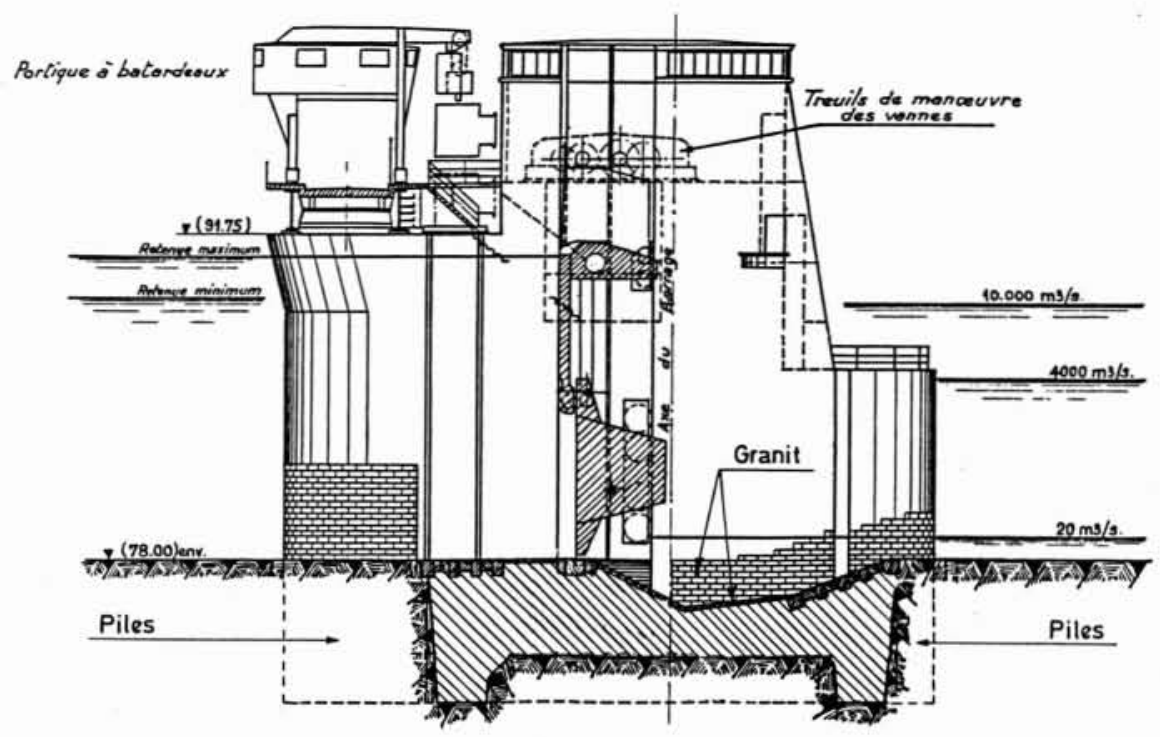

1. Baix-Logis-Neuf - Barrage de retenue. Coupe transversale.

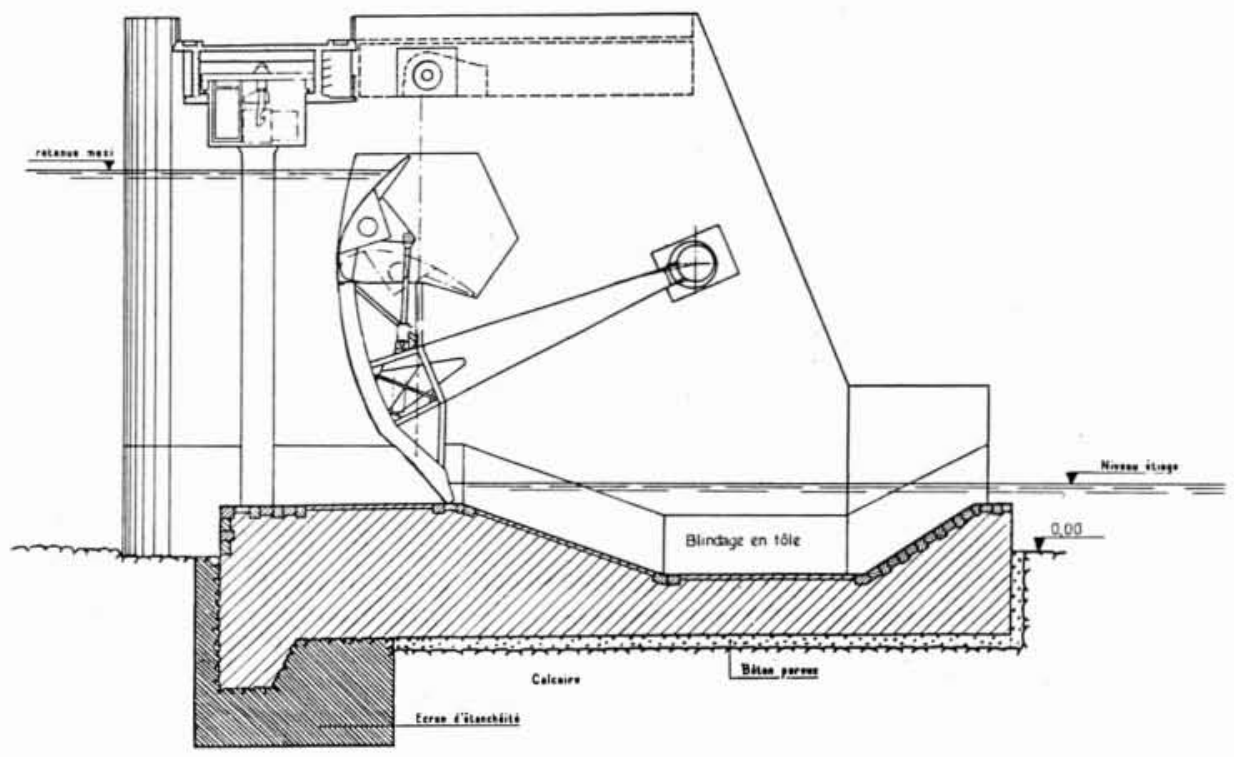

2. Vallabrègues - Barrage de retenue. Coupe transversale.

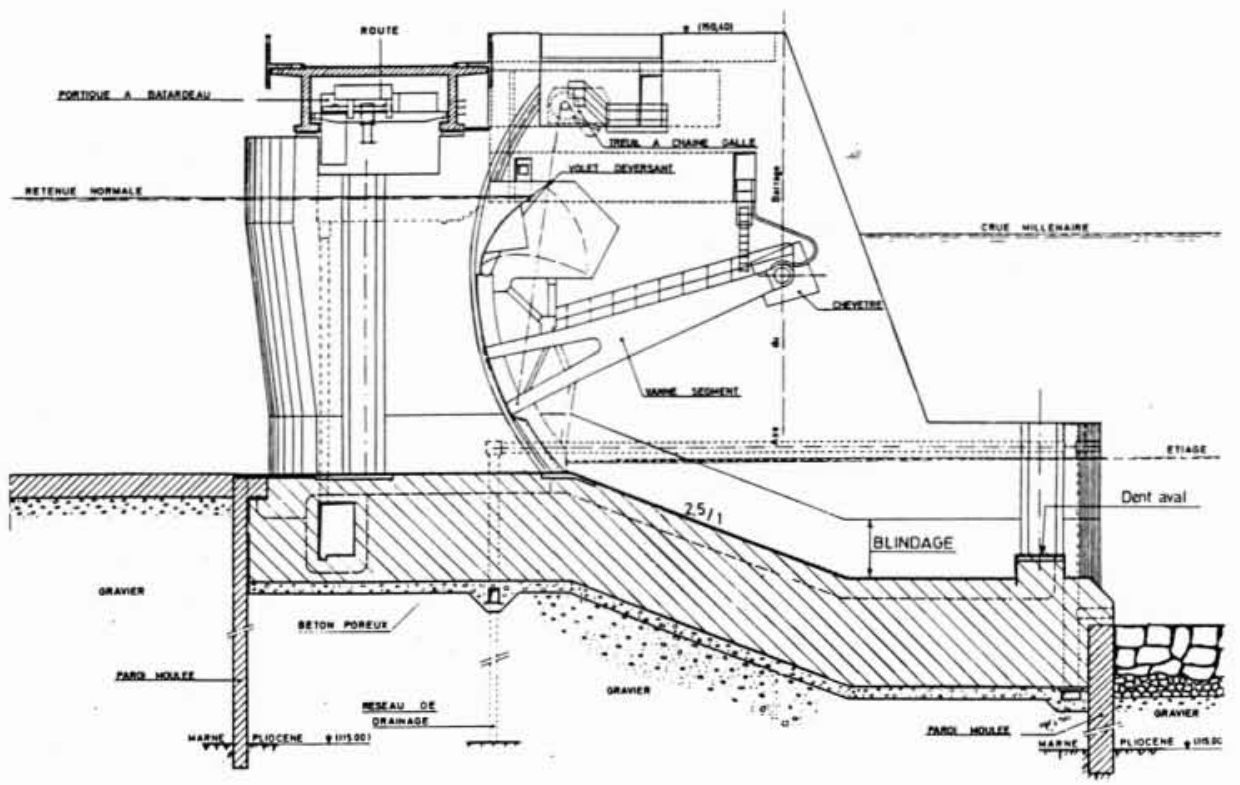

3. Péage de Roussillon - Barrage de retenue. Coupe transversale. 
corindon. Quant aux blindages, ils avaient été sensiblement réduits notamment dans les rainures à batardeau et sur les redans.

Dès la fin de l'année 1978 on notait d'importantes dégradations des deux murs en aile au niveau du plan d'eau. Une exploration subaquatique montrait une dégradation des redans, des rainures à batardeau et, à un moindre degré, des revêtements anti-usure des radiers.

Une étude sur modèle réduit réalisé au Laboratoire d'hydraulique de la Compagnie Nationale du Rhône à Gerland (Lyon), a montré que les dégradations avaient pour origine les courants de retour violents engendrés par la configuration des protections aval (enrochements) dont le profil était analogue à celui adopté au barrage de Caderousse mais dans un substratum de qualité différente (Marne). De plus, des calculs de déperdition d'énergie montraient que le barrage de Saint-Pierre-de-Bœuf était l'un de ceux qui dissipait la plus grande énergie et ceci dans des conditions hydrologiques relativement fréquentes.

Une première réparation a donc été entreprise à l'abri d'un batardeau en graviers, barrant entièrement le vieux Rhône, situé à une centaine de mètres à l'aval du barrage. Ces dispositions ont permis de réparer non seulement les dégâts apparus sur le radier lui-même, mais également de modifier complètement les protections en enrochements qui étaient en grande partie à la source de tous les ennuis. Sur l'ouvrage lui-même, les rainures aval ont été obturées dans les passes d'extrémité, afin de supprimer les rouleaux générateurs de dégradations dans ces zones particulièrement exposées. Les autres parties abîmées ont été réparées au moyen de mortier de résine sans autres précautions particulières, les nouvelles dispositions adoptées pour l'ensemble des protections aval paraissant très sûres.

Or en 1984, après trois années de fortes crues, d'importantes dégradations étaient de nouveau constatées notamment sur la face aval des redans, des blocs étant repérés sur le dessus des redans et sur le radier lui-même. Une nouvelle étude sur modèle réduit faisait ressortir que certains blocs mis en place lors de la réparation précédente avaient été arrachés et pris dans le rouleau créé à l'aval de l'ouvrage lors des déversements.

Il était donc décidé d'une part de percoler au béton les enrochements sur toute la partie amont du tapis de protection, de modifier ce dernier au raccordement avec le barrage, et d'autre part de procéder à de nouvelles réparations rendues nécessaires sur l'ouvrage lui-même. Des travaux plus importants s'imposaient donc et pour cela il était exclu de recourir au même procédé que celui adopté précédemment (batardeau barrant tout le vieux Rhône) et qui interdisait l'utilisation du barrage en cas de crue.

C'est pourquoi fut prise la décision de réaliser un système de batardement n'obturant qu'une passe à la fois, laissant ainsi les autres passes, libres de jouer leur rôle d'évacuateur de crues, et situé juste en aval des redans, afin d'isoler non seulement le redan lui-même mais également toute la partie aval du radier, les dégradations constatées touchant l'ensemble de ce dernier.

Les caractéristiques de ce batardeau devaient respecter les conditions suivantes :
- pouvoir s'appliquer successivement à toutes les passes du barrage;

- être mis en place facilement en cas de nécessité ultérieure ;

- pouvoir s'adapter à d'autres barrages aux caractéristiques voisines.

\section{Solution adoptée pour le batardement}

Plusieurs solutions ont fait l'objet d'analyses sommaires et d'évaluations globales permettant de les comparer. Ce sont les suivantes :

- poutres caissons flottantes;

- batardeau flottant ;

— batardeau à aiguilles en aluminium.

Le premier dispositif se compose de 3 groupes de 2 poutres caissons identiques, étanches, compartimentées, pouvant flotter de façon stable sur leur âme inférieure, puis échouées sur le lieu de batardement. Les poutres comportent des vannettes et évents permettant le ballastage par remplissage et le renflouement à l'air comprimé.

Le deuxième dispositif comporte des poutres identiques et de même conception que celles décrites à la solution précédente, mais ces poutres, qui peuvent flotter tant en position horizontale qu'en position verticale, sont assemblées pour constituer un tablier pouvant également flotter en position horizontale et en position verticale. Le tablier ainsi constitué, est halé par flottaison horizontale jusqu'à proximité de la passe à batardeau, amené en position verticale puis échoué sur le lieu de batardement.

Le troisième dispositif est composé de profilés spéciaux en aluminium s'appuyant en pied sur un seuil fixe, et en tête sur une poutre métallique démontable.

Les analyses ont montré que les deux premières solutions conduisaient à une adaptation plus délicate aux différents barrages concernés et que de plus elles étaient d'un coût supérieur.

C'est donc la solution « batardeau à aiguilles en aluminium " qui a été retenue pour une mise au point complète.

Il a été nécessaire tout d'abord de définir le « cahier des charges » de ce batardeau et notamment la hauteur des aiguilles compte tenu des conditions d'exploitation du barrage.

De plus, il était souhaité qu'il puisse s'adapter à un certain nombre d'autres ouvrages présentant des caractéristiques pas trop éloignées.

Il faut observer que si des batardeaux massifs mis en place dans des rainures appropriées ou des batardeaux précaires en maçonnerie peuvent s'accommoder d'une immersion occasionnelle soit parce qu'ils résisteront sûrement, soit parce qu'ils peuvent être rétablis dans de bonnes conditions après abaissement des eaux, il n'en est pas de même avec les batardeaux à aiguilles qui ne peuvent être immergés sans risque de perte irrémédiable entraînant nécessairement un réapprovisionnement long et onéreux. Il convenait donc de se garantir en prenant une certaine 

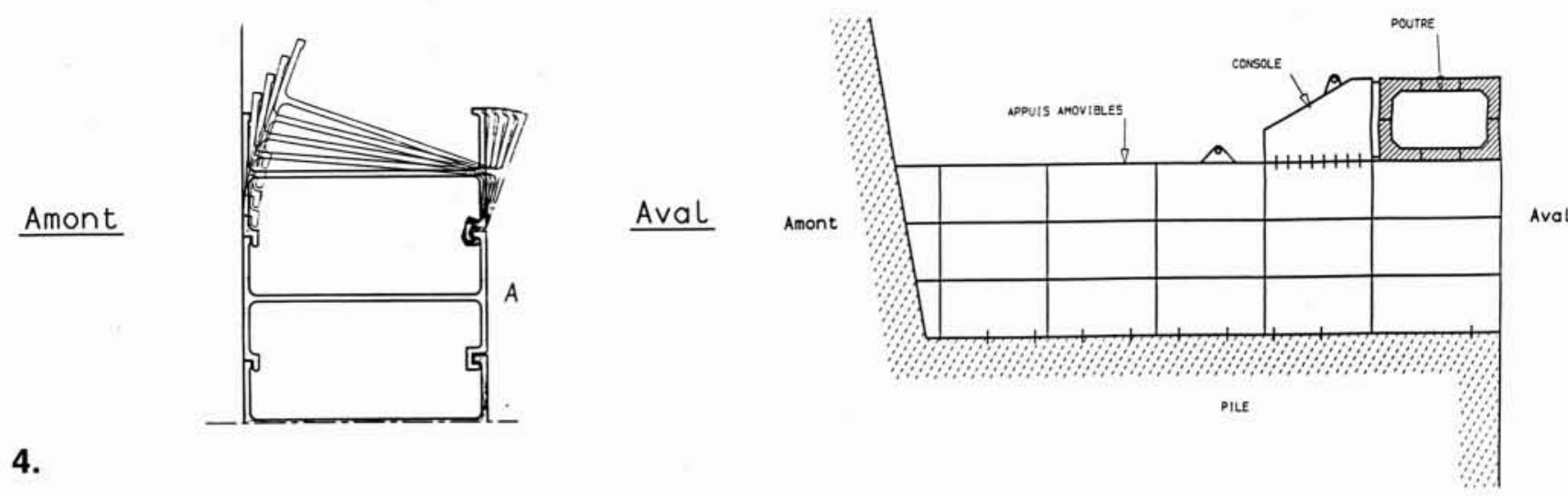

4.

6.

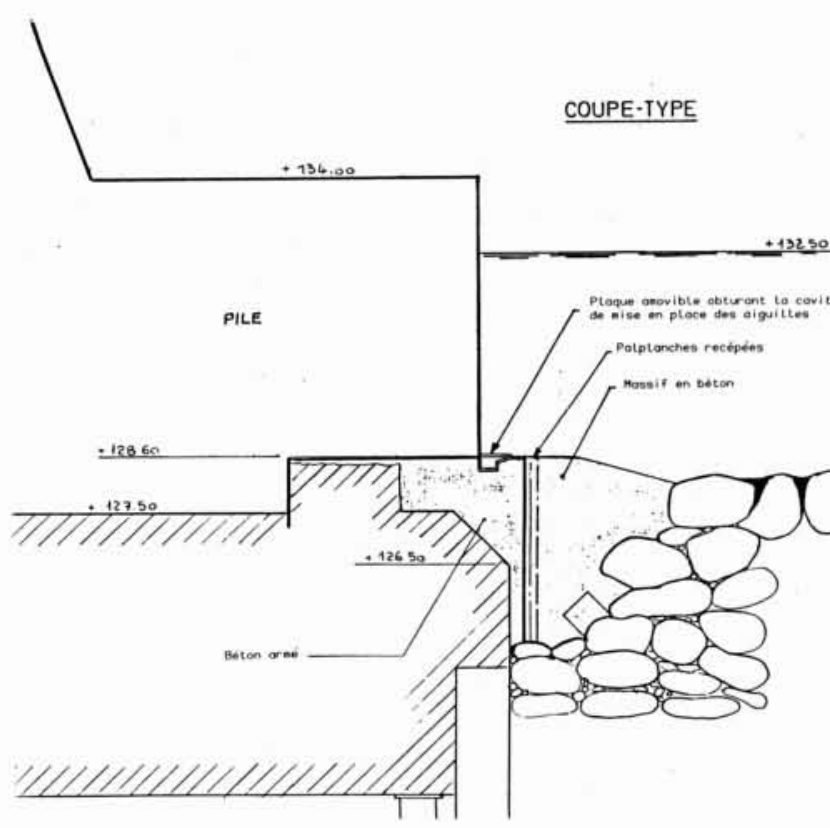

Péage de Roussillon - Barrage de retenue - Batardement à aiguilles.

5.

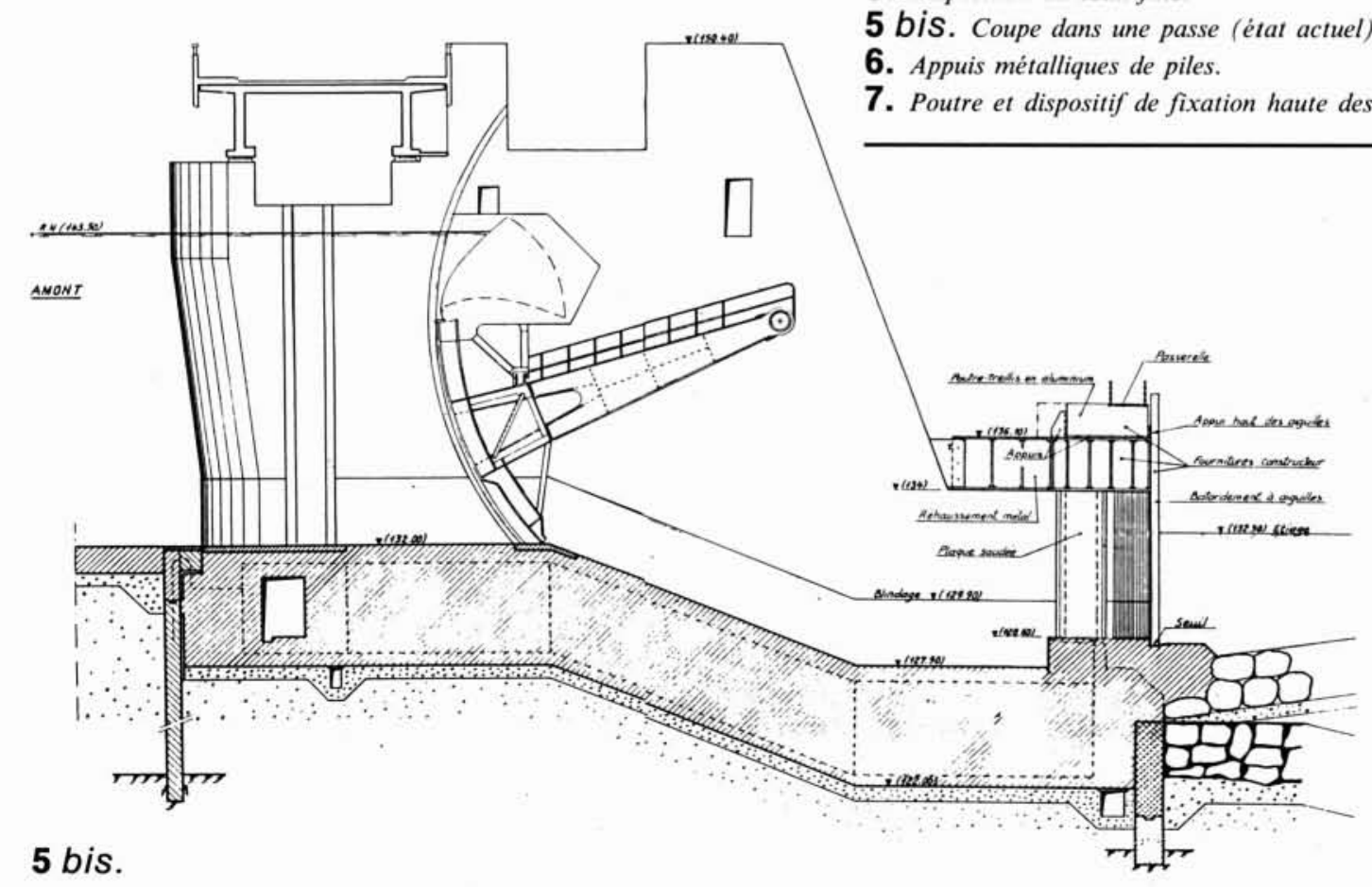

4. Enclenchement des aiguilles.

5. Disposition du seuil fixe.

5 bis. Coupe dans une passe (état actuel).

6. Appuis métalliques de piles.
7. Poutre et dispositif de fixation haute des aiguilles.

Aval

7.

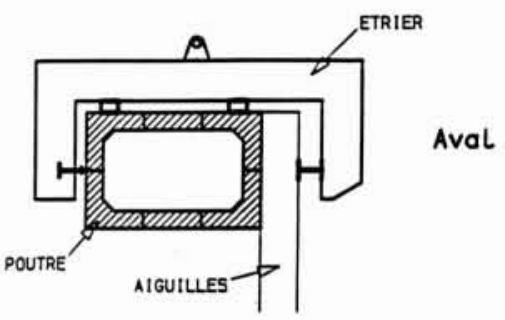


marge sur la cote d'arasement des aiguilles. La cote adoptée correspond au niveau existant à l'aval du barrage lors d'un débit semi-permanent du Rhône en supposant l'arrêt total de l'usine, le débit passant totalement par les autres passes non obturées du barrage.

\section{Dispositif adopté pour le batardement}

Le dispositif de batardement prévu consiste à réaliser un rideau avec des aiguilles en aluminium s'emboîtant les unes dans les autres (fig. 4). Les aiguilles s'appuyent, en partie basse, sur un seuil fixe scellé à l'extrémité aval des radiers du barrage et en partie haute, sur une poutre métallique démontable disposée entre les deux piles délimitant la passe à batarder. Le seuil est intégré dans le blindage de la dent (fig. 5 et 5 bis). Il est réalisé en tôle pliée. Compte tenu des conditions particulières du site et des considérations exposées ci-avant les aiguilles ont une longueur de $9,50 \mathrm{~m}$.

La poutre haute est une poutre-caisson en acier d'un poids total d'une vingtaine de tonnes. Elle repose sur les arrière-becs du barrage par l'intermédiaire d'appuis métalliques amovibles permettant d'augmenter de $2 \mathrm{~m}$ la protection contre les submersions (fig. 6). Pour les autres barrages concernés ces appuis métalliques seront adaptés suivant la hauteur à compenser. Les aiguilles sont disposées verticalement. Pour assurer leur stabilité, elles sont bloquées dans la rainure du seuil à l'aide de cales et rendues solidaires de la poutre haute par un dispositif d'étrier (fig. 7).

La poutre haute est mise en place sur les arrière-becs, soit par deux grues disposées sur le pont de service du barrage, soit par des mâts de levage à partir d'un ponton flottant. Les aiguilles sont installées à l'aide d'une grue placée sur le pont de service, l'enclenchement étant réalisé manuellement à partir de la poutre qui forme passerelle. Il est possible de manipuler jusqu'à cinq aiguilles à la fois sans problème.

L'étanchéité du rideau est assurée par remplissage, au mâchefer, des vides entre les aiguilles. Pour refermer le rideau d'aiguilles sur les arrière-becs ou sur les piles culées, les aiguilles de contact sont équipées de profilés caoutchouc type «note de musique».

\section{Déroulement de l'opération de batardement}

Deux méthodes peuvent être employées : manutention des pièces avec des engins terrestres ou avec un ponton flottant. Elles ont été testées en 1987 sur une passe de rive $\left(\mathrm{n}^{\circ} 1\right.$ de RG) et sur une passe courante $\left(n^{\circ} 5\right)$ du barrage de retenue de l'aménagement du Péage de Roussillon.

\section{Emploi de moyens terrestres (fig. 8)}

Le test a été effectué sur la passe 1 de rive gauche. La manutention de la poutre-caisson et de ses appuis- rehausses amovibles a nécessité l'utilisation de deux grues de $125 \mathrm{t}$. Celle des aiguilles en aluminium et de leur système de blocage s'est faite à l'aide d'une grue de $45 \mathrm{t}$.

Le déroulement de l'essai a été le suivant :

1) poutre-caisson :

- mise en place sur le pont de service des deux grues de $125 \mathrm{t}$ de part et d'autre de la poutre (longueur $26,5 \mathrm{~m}$, poids $22,5 \mathrm{t}$ ),

- mise en place à la grue de l'appui-rehausse amovible de pile (hauteur $2 \mathrm{~m}$, poids $6,9 \mathrm{t}$ ),

- pose provisoire de la poutre-caisson sur l'arrière du sommet des piles,

- mise en place de l'appui-rehausse de culée (hauteur $2 \mathrm{~m}$, poids $1,7 \mathrm{t}$ ) à l'aide d'une grue déplacée sur la plateforme aval,

- reprise de la poutre-caisson par les deux grues et pose sur l'appui de culée, et, en limite de portée de grue, sur l'appui de pile. Ripage sur cet appui de pile de la poutre jusqu'à sa position définitive,

- montage sur la poutre-caisson des garde-corps pour le personnel.

2) batardeau à aiguilles:

160 aiguilles en aluminium ont été approvisionnées sur le site ; 4 d'entre elles étaient munies d'un dispositif d'étanchéité réglable, utilisant un joint type " note de musique ". En forme de I, elles ont les caractéristiques suivantes : poids $152 \mathrm{~kg}$, hauteur $9,5 \mathrm{~m}$, âme $17 \mathrm{~cm}$, largeur $32 \mathrm{~cm}$, épaisseur $8 \mathrm{~mm}$. Posées et bloquées en pied sur leur seuil, elles doivent être plaquées en haut contre la poutre-caisson par des éléments de poutrelle I.P.N. soutenus et pincés par 12 étriers (poids variant de 373 à $418 \mathrm{~kg}$ suivant la position sur la poutre).

La mise en place des aiguilles s'est faite de la manière suivante :

- en partie basse :

- enlèvement sous l'eau des éléments de couvercles de protection du seuil d'appui des aiguilles, et nettoyage à la suceuse de ce dernier.

- en partie haute :

- mise en place du $1^{\text {er }}$ étrier sur la poutre (contre la culée),

- à l'aide de la grue de $45 \mathrm{t}$, mise en place de la première aiguille (celle de rive gauche munie d'un joint d'étanchéité). Blocage du pied de l'aiguille dans le seuil d'appui par une cale mise en place par un plongeur,

- pose, suivant le même scénario, des aiguilles suivantes. Toutes les 12 aiguilles, mise en place d'un élément recépé permettant le passage de l'étrier, pose de l'étrier, de l'élément de poutrelle pour bloquer les aiguilles déjà en place, - achèvement de l'opération par la mise en place de l'aiguille de rive droite munie d'un joint d'étanchéité.

Le démontage du batardeau s'est fait suivant le schéma inverse. A la fin de l'opération, le seuil d'appui a été nettoyé à la suceuse, les couvercles de protection reposés, boulonnés et cachetés au ciment prompt par un plongeur. 

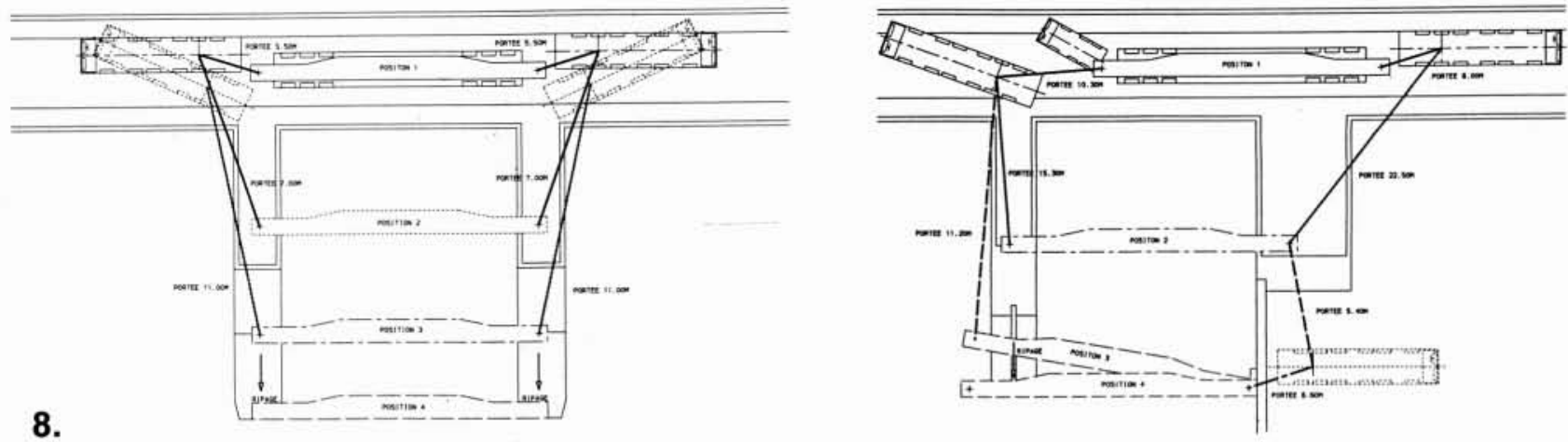

L'opération de batardement a duré 3 jours $(1 / 2$ journée pour la mise en place des appuis, 1 journée pour celle de la poutre-caisson, et 1 journée $1 / 2$ pour celle du batardeau à aiguilles). Le montage sur une autre passe nécessite la même durée. Il en est de même du démontage.

\section{Emploi de moyens fluviaux (fig. 9)}

La mise en place et l'enlèvement de la poutre-caisson à l'aide d'un ponton équipé de deux biguettes a été testée. C'est sur la passe courante $\mathrm{n}^{\circ} 3$ que l'essai s'est déroulé. Le ponton, ballasté pour rester équilibré lors des manutentions, était équipé de deux mâts d'élévation de 12,6 t.

L'appui de pile 4 ayant été mis en place au début du test terrestre, le déroulement de l'essai a été le suivant :

- levage de la poutre avec les biguettes et pose sur le ponton;

- déplacement du ponton de la passe 1 à la passe 5 et dépose provisoire de la poutre sur les arrière-becs de la passe 5 ;

- nouveau déplacement du ponton pour récupérer l'appui de pile et l'amener de la pile 1 à la pile 3 ;

- reprise de la poutre pour l'amener passe 3 sur ses appuis-rehausses, la régler et la bloquer.

Après cet essai de mise en place, la poutre-caisson a été enlevée comme suit :

- reprise sur le ponton ;

- déplacement du ponton vers la culée RG ;

- enlèvement de la poutre à la grue de $45 \mathrm{t}$ et stockage.

La durée de l'opération fluviale a été identique à celle utilisant les moyens terrestres.

\section{Comportement du batardeau}

A l'issue du test terrestre, la passe a été vidangée pour contrôler la résistance mécanique du batardeau et son étanchéité. Le dispositif de pompage comprenait 3 pompes capables d'évacuer $1400 \mathrm{~m}^{3} / \mathrm{h}$ et de vider l'enceinte en $1 / 2$ journée. L'étanchéité s'étant révélée insuffisante, les alvéoles des aiguilles en aluminium ont été remplies avec du mâchefer.

Ainsi, le débit d'exhaure a pu être réduit à $300 \mathrm{~m}^{3} / \mathrm{h}$ et l'auge vidée dans les conditions prévues.

L'essai ayant été satisfaisant, le pompage a été arrêté.
Péage de Roussillon - Barrage de retenue - Batardement à aiguilles.

8. Mise en place de la poutre caisson avec des grues. A gauche: passe courante.

A droite: passe de rive.

9. Mise en place de la poutre caisson avec un ponton flottant.

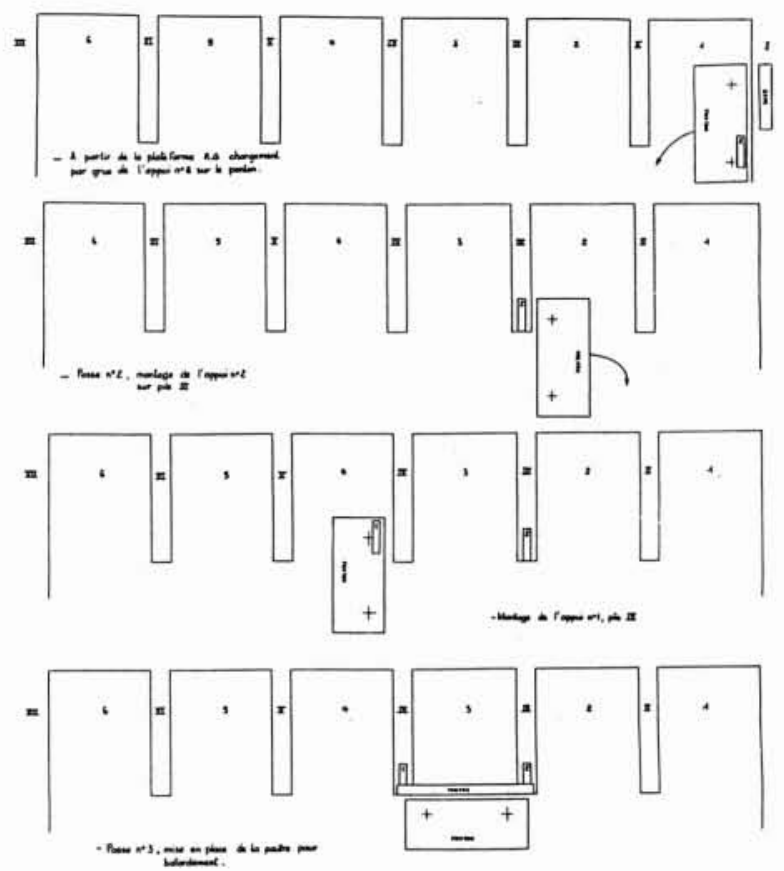

9.

\section{Conclusion}

Le test fait au barrage de l'aménagement du Péage de Roussillon a montré que ce système de batardeau à aiguilles, bien que d'une mise en place délicate mais relativement rapide, a donné satisfaction. Il peut être utilisé sur plusieurs barrages de dimensions voisines, et permet de pallier l'absence de batardeaux aval classiques. Le batardement avec des aiguilles légères est le système le plus pratique et le mieux adapté pour ce type de situation. 